\title{
A new family of anisotropic zinc-based semiconductors in a shallow energy landscape
}

\author{
Supporting Information
}

\begin{abstract}
Ankita Bhutani ${ }^{\dagger}$ Xiao Zhang, ${ }^{\dagger}$ Piush Behera, Rangarajan Thiruvengadam, Shannon E.
\end{abstract}
Murray, André Schleife, Daniel P. Shoemaker

\section{Convex hull}

In the presentation of calculated convex hulls, we evaluate stability of $A-\mathrm{Zn}-Q$ ternaries from the DFT-PBE total energies relative to the binaries. The energy $\Delta E$ is generated by normalizing the two binaries $X=A_{2} Q$ and $Y=Z n Q$ to zero energy, with:

$$
E_{\mathrm{hull}}^{X_{\alpha} Y_{1-\alpha}}=E_{\mathrm{tot}}^{X_{\alpha} Y_{1-\alpha}}-\alpha E_{\mathrm{tot}}^{X}-(1-\alpha) E_{\mathrm{tot}}^{Y}
$$

In the equation, $E_{\text {tot }}^{X_{\alpha} Y_{1-\alpha}}, E_{\text {tot }}^{X}$, and $E_{\text {tot }}^{Y}$ are the total energies of the ternaries, the total energies of the binary end components $X$, and the total energies of the binary end components $Y$, respectively. In our DFT simulations, a total number of 46 compounds are examined, and these are picked according to the probabilities given by the Structure Predictor tool from the Materials Project. [1, 2] In order to obtain the formation energy, i.e. the last column in Table 1 in the main text, we calculated the total energy for the elements: $E_{K}=-1.0530$ $\mathrm{eV} /$ atom, $E_{N a}=-1.3113 \mathrm{eV} /$ atom, $E_{Z n}=-1.1105 \mathrm{eV} /$ atom, $E_{S}=-3.4644 \mathrm{eV} /$ atom and $E_{S e}=-3.5058 \mathrm{eV} /$ atom. For sulfides, a correction of $-0.6635 \mathrm{eV} /$ atom to the total energy of the compounds is applied when calculating the formation energy $\Delta H^{f}$, as suggested by the Materials Project[2]. We verified that the calculated formation energies shown in Table 1 in the main text agrees within $0.005 \mathrm{eV}$ /atom, whichever data is available from the Materials Project[2]. 
Table S1: DFT-PBE total energies $E$ for Na-containing compounds. The stoichiometry follows the notation of $A-\mathrm{Zn}-Q$. For equivalent compositions where different structures are studied, the different structures are distinguished with numbering in square brackets. The k-point samplings used are listed in parentheses. $V_{\text {cell }}$ and $E_{\text {tot }}$ are the optimized volume and total energy of the unit cell, and $N$ is the number of atoms per unit cell.

\begin{tabular}{lllllll}
\hline Stoichiometry & Space Group & $V_{\text {cell }}\left(\AA^{3}\right)$ & $E_{\text {tot }}(\mathrm{eV})$ & $N$ & $\Delta E(\mathrm{eV} /$ atom $)$ & Observed? \\
\hline Na-Zn-S & & & & & & \\
$2-0-1(8 \times 8 \times 8)$ & $F m \overline{3} m$ & 70.93 & -9.976 & 3 & 0.000 & + \\
$6-1-4(2 \times 2 \times 3)$ & $C m c 2_{1}$ & 484.86 & -74.042 & 22 & -0.015 & \\
$2-1-2[1](4 \times 2 \times 4)$ & Ibam & 216.13 & -33.805 & 10 & -0.005 & \\
$2-1-2[2](3 \times 4 \times 4)$ & $P 2_{1} / c$ & 436.68 & -67.752 & 20 & -0.012 & \\
$2-1-2[3](1 \times 3 \times 3)$ & $P b c a$ & 908.95 & -134.349 & 40 & 0.017 & \\
$4-3-5(1 \times 5 \times 1)$ & $P n m a$ & 979.36 & -162.822 & 48 & -0.007 & \\
$2-2-3[1](2 \times 2 \times 4)$ & $C 2 / c$ & 589.35 & -95.226 & 28 & -0.009 & + \\
$2-2-3[2](4 \times 4 \times 4)$ & $P 2_{1} / c$ & 308.60 & -47.067 & 14 & 0.030 & \\
$2-2-3[3](4 \times 4 \times 2)$ & $P 4_{3} 2_{1} 2$ & 613.72 & -94.167 & 28 & 0.029 & \\
$2-3-4(3 \times 4 \times 2)$ & $P 2 / c$ & 397.73 & -60.092 & 18 & 0.062 & + \\
$0-1-1(8 \times 8 \times 8)$ & $F 43 m$ & 40.43 & -6.851 & 2 & 0.000 & + \\
\hline Na-Zn-Se & & & & & & + \\
$2-0-1(8 \times 8 \times 8)$ & $F m \overline{3} m$ & 80.47 & -9.342 & 3 & 0.000 & + \\
$6-1-4(2 \times 2 \times 3)$ & $C m c 2_{1}$ & 552.66 & -68.429 & 22 & -0.021 & + \\
$4-1-3(3 \times 4 \times 4)$ & $P 1$ & 452.17 & -48.957 & 16 & 0.021 & + \\
$10-4-9(3 \times 3 \times 2)$ & $P \overline{1}$ & 1306.30 & -140.383 & 46 & 0.018 & \\
$2-1-2[1](4 \times 2 \times 4)$ & $I b a m$ & 245.53 & -30.839 & 10 & -0.019 & + \\
$2-1-2[2](3 \times 4 \times 4)$ & $P 2_{1} / c$ & 497.37 & -61.758 & 20 & -0.023 & \\
$4-3-5(1 \times 5 \times 1)$ & $P n m a$ & 1127.39 & -147.529 & 48 & -0.019 & \\
$2-2-3[1](4 \times 4 \times 4)$ & $P 2_{1} / c$ & 357.22 & -42.331 & 14 & 0.025 & \\
$2-2-3[2](4 \times 4 \times 2)$ & $P 4_{3} 2_{1} 2$ & 673.59 & -84.737 & 28 & 0.022 & \\
$2-2-3[3](2 \times 2 \times 3)$ & $C 2 / c$ & 677.92 & -85.865 & 28 & -0.018 & \\
$2-3-4[1](4 \times 4 \times 4)$ & Ibam & 438.88 & -53.824 & 18 & 0.050 & \\
$2-3-4[2](3 \times 4 \times 2)$ & $P 2 / c$ & 438.34 & -53.853 & 18 & 0.048 & + \\
$0-1-1(8 \times 8 \times 8)$ & $F \overline{4} 3 m$ & 47.28 & -6.031 & 2 & 0.000 & \\
& & & & & &
\end{tabular}


Table S2: DFT-PBE total energies $E$ for K-containing compounds. The stoichiometry follows the notation of $A-Z n-Q$. For equivalent compositions where different structures are studied, the different structures are distinguished with numbering in square brackets. The k-point samplings used are listed in parentheses. $V_{\text {cell }}$ and $E_{\text {tot }}$ are the optimized volume and total energy of the unit cell, and $N$ is the number of atoms per unit cell.

\begin{tabular}{lllllll} 
Stoichiometry & Space Group & $V_{\text {cell }}\left(\AA^{3}\right)$ & $E_{\text {tot }}(\mathrm{eV})$ & $N$ & $\Delta E(\mathrm{eV} /$ atom $)$ & Observed? \\
\hline K-Zn-S & & & & & & \\
$2-0-1(8 \times 8 \times 8)$ & $F m \overline{3} m$ & 104.29 & -9.421 & 3 & 0.000 & + \\
$6-1-4[1](2 \times 2 \times 3)$ & $P 6_{3} m c$ & 653.33 & -71.472 & 22 & -0.037 & + \\
$6-1-4[2](3 \times 3 \times 5)$ & $P 4_{2} / n m c$ & 684.92 & -68.971 & 22 & 0.077 & \\
$4-1-3(2 \times 2 \times 3)$ & $P \overline{1}$ & 978.28 & -104.086 & 32 & -0.017 & \\
$6-2-5(3 \times 3 \times 2)$ & $P 4_{2} / n m c$ & 863.56 & -84.846 & 26 & -0.009 & \\
$2-1-2[1](4 \times 2 \times 4)$ & Ibam & 270.73 & -33.597 & 10 & -0.077 & + \\
$2-1-2[2](2 \times 4 \times 1)$ & $P 2_{1} / c$ & 1234.91 & -133.009 & 40 & -0.042 & \\
$2-1-2[3](2 \times 3 \times 3)$ & $P b c m$ & 621.62 & -65.149 & 20 & 0.026 & \\
$4-3-5[1](1 \times 5 \times 1)$ & $P n m a$ & 1264.39 & -158.611 & 48 & 0.007 & \\
$2-2-3[1](4 \times 3 \times 3)$ & $P 2 / c$ & 350.94 & -46.726 & 14 & -0.007 & \\
$2-2-3[2](2 \times 2 \times 4)$ & $C 2 / c$ & 684.92 & -94.137 & 28 & -0.031 & \\
$2-3-4[1](1 \times 2 \times 3)$ & $P n m a$ & 854.41 & -122.036 & 36 & -0.036 & \\
$2-3-4[2](3 \times 4 \times 2)$ & $P 2 / c$ & 427.31 & -61.071 & 18 & -0.039 & + \\
$2-3-4[3](3 \times 4 \times 4)$ & $P \overline{1}$ & 458.34 & -59.515 & 18 & 0.048 & \\
$2-3-4[4](8 \times 4 \times 2)$ & $F d d d$ & 471.48 & -55.450 & 18 & 0.274 & \\
$2-6-7[1](2 \times 2 \times 7)$ & $P 4_{2} n m$ & 668.39 & -100.837 & 30 & 0.024 & \\
$0-1-1(8 \times 8 \times 8)$ & $F \overline{4} 3 m$ & 40.43 & -6.851 & 2 & 0.000 & + \\
\hline K-Zn-Se & & & & & & \\
$2-0-1(8 \times 8 \times 8)$ & $F m \overline{3} m$ & 116.59 & -8.918 & 3 & 0.000 & + \\
$6-1-4(2 \times 2 \times 3)$ & $P 6_{3} m c$ & 735.18 & -66.720 & 22 & -0.049 & \\
$4-1-3(2 \times 2 \times 3)$ & $P \overline{1}$ & 1053.25 & -96.469 & 32 & -0.028 & \\
$2-1-2[1](2 \times 4 \times 1)$ & $P 2_{1} / c$ & 1256.16 & -121.114 & 40 & -0.034 & + \\
$2-1-2[2](6 \times 3 \times 6)$ & Ibam & 305.60 & -30.891 & 10 & -0.095 & + \\
$4-3-5[1](1 \times 5 \times 1)$ & $P n m a$ & 1347.49 & -144.328 & 48 & -0.009 & \\
$2-2-3[1](2 \times 2 \times 3)$ & $C 2 / c$ & 781.98 & -85.403 & 28 & -0.049 & \\
$2-2-3[2](4 \times 3 \times 3)$ & $P 2_{1} / c$ & 404.09 & -42.257 & 14 & -0.017 & \\
$2-3-4[1](3 \times 4 \times 2)$ & $P 2 / c$ & 485.07 & -55.054 & 18 & -0.054 & + \\
$2-3-4[2](1 \times 2 \times 3)$ & $P n m a$ & 974.39 & -109.911 & 36 & -0.048 & \\
$2-3-4[3](3 \times 3 \times 4)$ & $P \overline{1}$ & 518.69 & -54.331 & 18 & -0.014 & \\
$2-6-7(2 \times 2 \times 7)$ & $P 2$ & 774.43 & -89.783 & 30 & 0.017 & \\
$0-1-1(8 \times 8 \times 8)$ & $F \overline{4} 3 m$ & 47.28 & -6.031 & 2 & 0.000 & + \\
& & & & & & \\
& & &
\end{tabular}




\section{Experimental results}
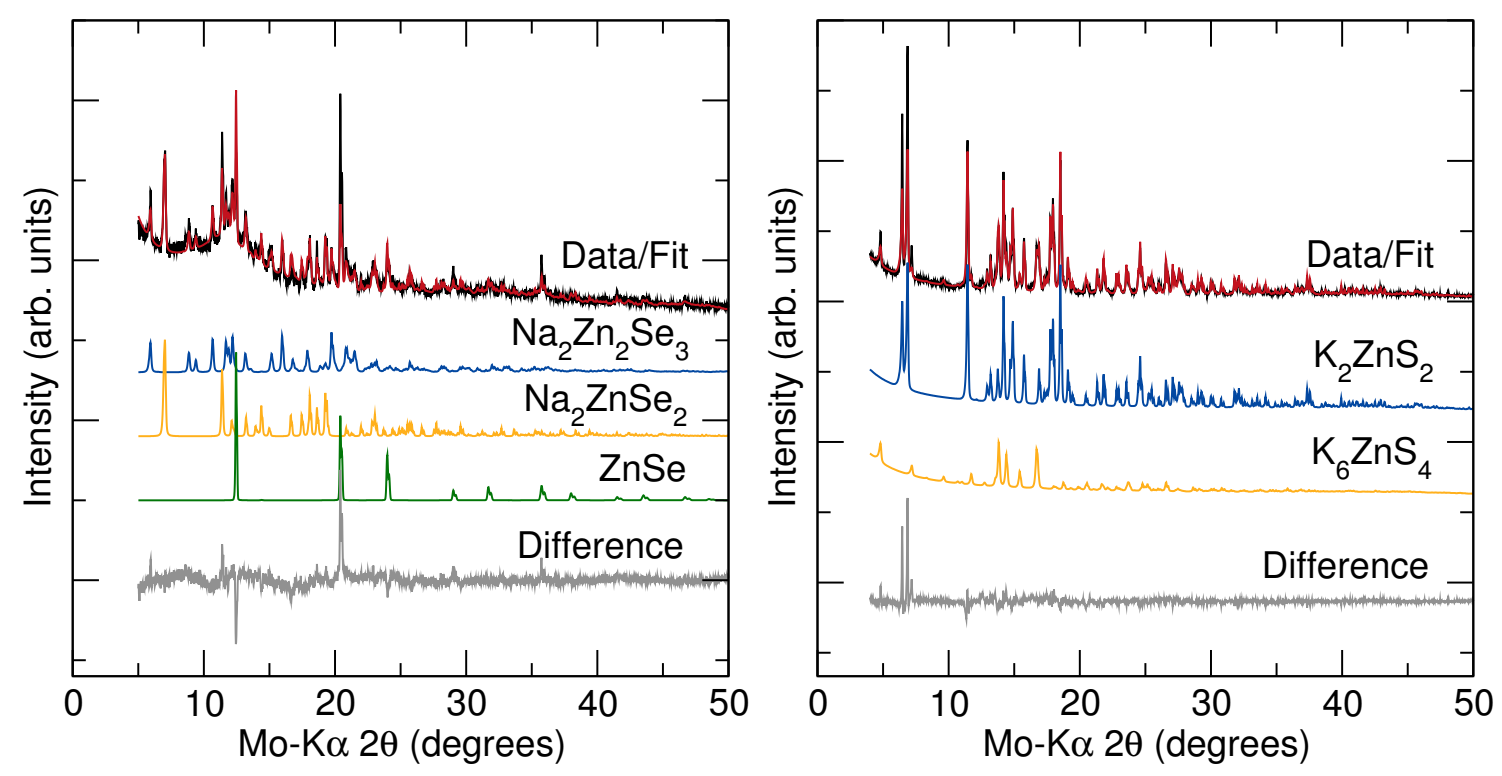

Figure S1: X-ray diffraction data and refinements for the minor impurity phases $\mathrm{Na}_{2} \mathrm{Zn}_{2} \mathrm{Se}_{3}$ (left, $C 2 / c, a=15.31 \AA, b=13.67 \AA, c=6.987 \AA, \beta=116.5^{\circ}$ ) and $\mathrm{K}_{6} \mathrm{ZnS}_{4}$ (right, $P 6_{3} m c$, $a=9.8 \AA, c=7.62 \AA)$. They belong to the same structure types $\left(\mathrm{Na}_{2} \mathrm{Mn}_{2} \mathrm{~S}_{3}\right.$ and $\mathrm{K}_{6} \mathrm{HgS}_{4}$, respectively) as the other phases reported here, but atomic positions are not reliably refined. 


\begin{tabular}{lllllll}
\hline Atom & Site & $\mathrm{x}$ & $\mathrm{y}$ & $\mathrm{z}$ & Occ. & $B_{e q}$ \\
\hline Na1 & $8 f$ & $0.040(3)$ & $0.962(3)$ & $0.130(6)$ & 1 & 2 \\
Na2 & $4 e$ & 0.0 & $0.688(4)$ & 0.25 & 1 & 2 \\
Na3 & $4 e$ & 0.0 & $0.425(4)$ & 0.25 & 1 & $3.5(8)$ \\
Zn1 & $8 f$ & $0.2119(11)$ & $0.5882(13)$ & $0.300(2)$ & 1 & 2 \\
Zn2 & $8 f$ & $0.1835(10)$ & $0.2702(10)$ & $0.277(3)$ & 1 & 2 \\
S1 & $8 f$ & $0.1370(18)$ & $0.409(3)$ & $0.105(4)$ & 1 & 2 \\
S2 & $8 f$ & $0.1440(19)$ & $0.7065(18)$ & $0.090(4)$ & 1 & 2 \\
S3 & $8 f$ & $0.1454(18)$ & $0.0247(19)$ & $0.0252(5)$ & 1 & 2 \\
\hline
\end{tabular}

Table S3: Atomic positions and displacement parameters of $\mathrm{Na}_{2} \mathrm{Zn}_{2} \mathrm{~S}_{3}$ at $300 \mathrm{~K}$. Monoclinic, $C 2 / c, Z=8, a=14.708(3) \AA, b=13.106(3) \AA, c=6.6656(11) \AA, \beta=116.161(13)^{\circ}$.

\begin{tabular}{lllllll}
\hline Atom & Site & $\mathrm{x}$ & $\mathrm{y}$ & $\mathrm{z}$ & Occ. & $B_{e q}$ \\
\hline Na1 & $6 c$ & $0.1487(6)$ & $-0.1487(6)$ & $0.5466(11)$ & 1 & 2 \\
Na2 & $6 c$ & $0.5329(5)$ & $-0.5329(5)$ & $0.373(2)$ & 1 & 2 \\
Zn1 & $2 b$ & 0.3333 & 0.6667 & 0.2500 & 1 & 2 \\
Se1 & $2 b$ & 0.3333 & 0.6667 & $0.6026(17)$ & 1 & 2 \\
Se2 & $6 c$ & $0.18661(16)$ & $-0.18661(16)$ & $0.1451(15)$ & 1 & 2 \\
\hline
\end{tabular}

Table S4: Atomic positions and displacement parameters of $\mathrm{Na}_{6} \mathrm{ZnSe}_{4}$ at $300 \mathrm{~K}$. Hexagonal, $P 6_{3} m c, Z=2, a=9.3106(11) \AA, c=7.1537(9) \AA$.

\begin{tabular}{lllllll}
\hline Atom & Site & $\mathrm{x}$ & $\mathrm{y}$ & $\mathrm{z}$ & Occ. & $B_{e q}$ \\
\hline Na1 & $8 j$ & $0.3496(12)$ & $0.1438(8)$ & 0.0 & 1 & 2 \\
Zn1 & $4 a$ & 0.0 & 0.0 & 0.25 & 1 & 2 \\
Se1 & $8 j$ & $0.2151(4)$ & $0.8848(2)$ & 0.0 & 1 & 2 \\
\hline
\end{tabular}

Table S5: Atomic positions and displacement parameters of $\mathrm{Na}_{2} \mathrm{ZnSe}_{2}$ at $300 \mathrm{~K}$. Orthorhombic, Ibam, $Z=4, a=6.6282(9) \AA, b=11.6830(17) \AA, c=6.1588(9) \AA$.

\begin{tabular}{lllllll}
\hline Atom & Site & $\mathrm{x}$ & $\mathrm{y}$ & $\mathrm{z}$ & Occ. & $B_{\text {eq }}$ \\
\hline K1 & $8 j$ & $0.3353(4)$ & $0.1458(2)$ & 0.0 & 1 & 2 \\
Zn1 & $4 a$ & 0.0 & 0.0 & 0.25 & 1 & 2 \\
S1 & $8 j$ & $0.1859(6)$ & $0.8989(3)$ & 0.0 & 1 & 2 \\
\hline
\end{tabular}

Table S6: Atomic positions and displacement parameters of $\mathrm{K}_{2} \mathrm{ZnS}_{2}$ at $300 \mathrm{~K}$. Orthorhombic, Ibam, $Z=4, a=6.7385(5) \AA, b=12.5784(8) \AA, c=6.1699(4) \AA$. 


\begin{tabular}{lllllll}
\hline Atom & Site & $\mathrm{x}$ & $\mathrm{y}$ & $\mathrm{Z}$ & Occ. & $B_{e q}$ \\
\hline K1 & $2 a$ & 0 & 0 & 0 & 1 & 2 \\
$\mathrm{~K} 2$ & 23 & 0 & $0.515(4)$ & 0.25 & 1 & 2 \\
$\mathrm{Zn} 1$ & $2 f$ & 0.5 & $0.723(4)$ & 0.2500 & 1 & 2 \\
Zn2 & $4 g$ & $0.493(5)$ & $0.241(4)$ & $0.0149(8)$ & 1 & 2 \\
$\mathrm{~S} 1$ & $4 g$ & $0.294(13)$ & $0.496(4)$ & $0.077(4)$ & 1 & 2 \\
S2 & $4 g$ & $0.300(13)$ & $0.020(3)$ & $0.321(4)$ & 1 & 2 \\
\hline
\end{tabular}

Table S7: Atomic positions and displacement parameters of $\mathrm{K}_{2} \mathrm{Zn}_{3} \mathrm{~S}_{4}$ at $300 \mathrm{~K}$. Monoclinic, $P 2 / c, Z=2, a=7.156(2) \AA, b=5.7218(9) \AA, c=10.8566(17) \AA, \beta=112.24(3)^{\circ}$.

\begin{tabular}{lllllll}
\hline Atom & Site & $\mathrm{x}$ & $\mathrm{y}$ & $\mathrm{z}$ & Occ. & $B_{e q}$ \\
\hline K1 & $8 j$ & $0.3315(6)$ & $0.1455(3)$ & 0.0 & 1 & 2 \\
Zn1 & $4 a$ & 0.0 & 0.0 & 0.25 & 1 & 2 \\
Se1 & $8 j$ & $0.1922(3)$ & $0.8950(1)$ & 0.0 & 1 & 2 \\
\hline
\end{tabular}

Table S8: Atomic positions and displacement parameters of $\mathrm{K}_{2} \mathrm{ZnSe}_{2}$. Orthorhombic, Ibam, $Z=4, a=6.9283(4) \AA, b=13.1135(8) \AA, c=6.4346(4) \AA$.

\begin{tabular}{lllllll}
\hline Atom & Site & $\mathrm{x}$ & $\mathrm{y}$ & $\mathrm{Z}$ & Occ. & $B_{e q}$ \\
\hline $\mathrm{K} 1$ & $2 d$ & 0.5 & 0.0 & 0.5 & 1 & 2 \\
$\mathrm{~K} 2$ & $2 f$ & 0.5 & $0.5135(67)$ & 0.25 & 1 & $2.0(12)$ \\
Zn1 & $2 e$ & 0.0 & $0.2358(67)$ & 0.2500 & 1 & $2.0(6)$ \\
Zn2 & $4 g$ & 0.01005 & $0.2750(31)$ & $0.9886(11)$ & 1 & $2.0(4)$ \\
Se1 & $4 g$ & $0.7881(30)$ & $0.5083(25)$ & $0.5717(17)$ & 1 & $1.835(33)$ \\
Se2 & $4 g$ & $0.7934(28)$ & $0.9879(18)$ & $0.8259(15)$ & 1 & 2 \\
\hline
\end{tabular}

Table S9: Atomic positions and displacement parameters of $\mathrm{K}_{2} \mathrm{Zn}_{3} \mathrm{Se}_{4}$ at $300 \mathrm{~K}$. Monoclinic, $P 2 / c, Z=2, a=7.4507(15) \AA, b=5.9184(8) \AA, c=11.3542(15) \AA, \beta=112.537(16)^{\circ}$. 


\section{Relaxation}

Structural relaxation was performed with DFT using the PBE exchange-correlation functional. The inputs were the experimentally-determined structures, converted to standard primitive cells [3] using the AFLOW package. [4] The primitive cell of the different compositions used in simulation is shown in Fig. S2. We verified that no change in the structure itself is induced by the conversion.

The structural relaxation results are shown in Table S10. All lattice parameters are shown after converting to standard primitive cells, except for the $A_{2} \mathrm{Zn} Q_{2}$ structure, which are shown for the standard conventional cell with orthogonal axes. The computationally-predicted lattice parameters agree very well with experimental measurements, with a maximum difference $<2 \%$. These relaxed structures are used to generate electronic and optical properties. The optical properties are calculated along Cartesian coordinates, and for all structures, the Cartesian coordinate of the lattice vectors follows the convention of Setyawan, [3] with the simulated primitive cells matching that for Hexagonal (HEX), Body-centered orthorhombic (ORCI), Monoclinic (MCL) and C-centered monoclinic (MCLC), respectively for $A_{6} \mathrm{Zn} Q_{4}$, $A_{2} \mathrm{Zn} Q_{2}, A_{2} \mathrm{Zn}_{3} Q_{4}$, and $A_{2} \mathrm{Zn}_{2} Q_{3}$ structures.

Table S10: Comparison between the relaxed structures from simulation, to experimental results presented in this work.

\begin{tabular}{c|ccccccccc}
\hline Structures & $a$ & $b$ & $c$ & Expt. $a$ & Expt. $b$ & Expt. $c$ & $\Delta_{a}(\%)$ & $\Delta_{b}(\%)$ & $\Delta_{c}(\%)$ \\
\hline $\mathrm{K}_{2} \mathrm{Zn}_{3} \mathrm{~S}_{4}$ & 5.784 & 7.234 & 10.659 & 5.723 & 7.156 & 10.856 & 1.063 & 1.091 & -1.813 \\
$\mathrm{~K}_{2} \mathrm{Zn}_{3} \mathrm{Se}_{4}$ & 6.006 & 7.543 & 11.152 & 5.918 & 7.451 & 11.354 & 1.491 & 1.229 & -1.782 \\
$\mathrm{~K}_{2} \mathrm{ZnS}_{2}$ & 6.258 & 6.801 & 12.726 & 6.170 & 6.738 & 12.578 & 1.425 & 0.940 & 1.176 \\
$\mathrm{~K}_{2} \mathrm{ZnSe}_{2}$ & 6.553 & 7.019 & 13.288 & 6.436 & 6.929 & 13.118 & 1.813 & 1.300 & 1.295 \\
$\mathrm{~K}_{6} \mathrm{ZnS}_{4}$ & 9.877 & 9.877 & 7.731 & 9.779 & 9.779 & 7.623 & 1.005 & 1.005 & 1.411 \\
$\mathrm{Na}_{2} \mathrm{ZnSe}_{2}$ & 6.226 & 6.713 & 11.735 & 6.159 & 6.628 & 11.683 & 1.089 & 1.285 & 0.447 \\
$\mathrm{Na}_{6} \mathrm{ZnSe}_{4}$ & 9.389 & 9.389 & 7.235 & 9.310 & 9.310 & 7.153 & 0.849 & 0.849 & 1.153 \\
$\mathrm{Na}_{2} \mathrm{Zn}_{2} \mathrm{~S}_{3}$ & 9.904 & 9.904 & 6.772 & 9.850 & 9.850 & 6.666 & 0.551 & 0.551 & 1.590 \\
$\mathrm{Na}_{2} \mathrm{Zn}_{2} \mathrm{Se}_{3}$ & 10.371 & 10.371 & 7.127 & - & - & - & - & - & - \\
\hline
\end{tabular}



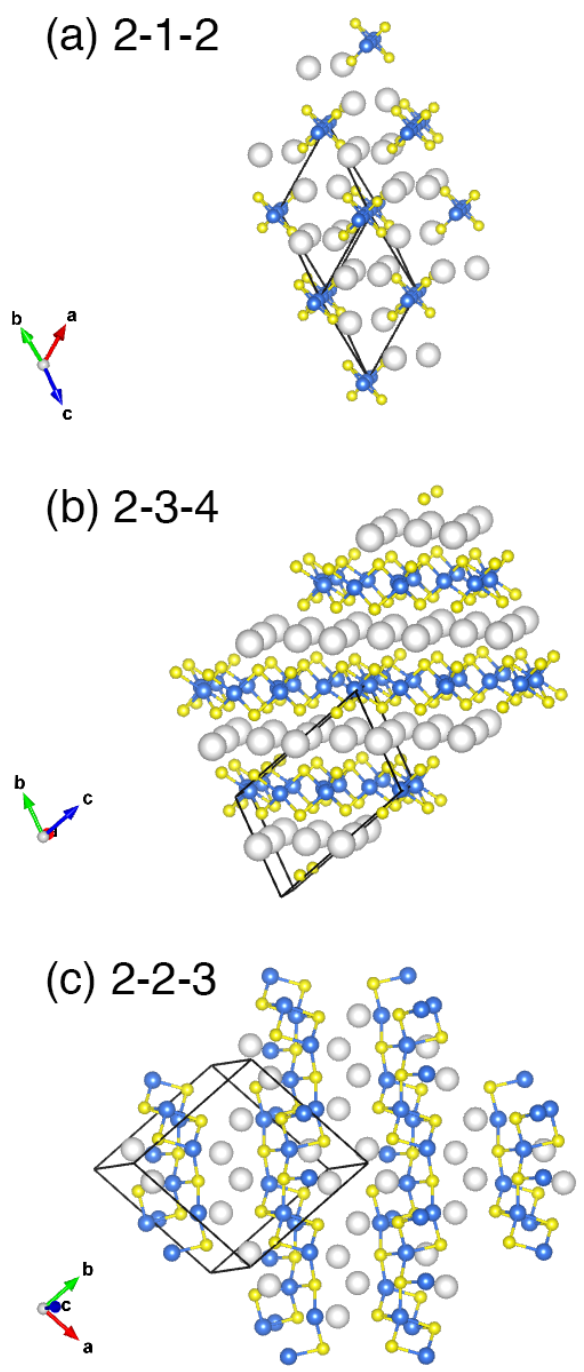

Figure S2: The standard primitive cell [3] of (a) $A_{2} \mathrm{Zn} Q_{2}$, (b) $A_{2} \mathrm{Zn}_{3} Q_{4}$, and (c) $A_{2} \mathrm{Zn}_{2} Q_{3}$, under the same view as in the main text and the same color scheme: Alkali metal, zinc, and chalcogen are shown in white, blue, and yellow, respectively. The $A_{6} \mathrm{Zn} Q_{4}$ structures are the same as that in the main text, thus are not shown again here. 


\section{Electronic band structures}

Here we show the electronic band gaps calculated using both the DFT-PBE and the hybrid exchange-correlation functionals. [5, 6] All of the band gaps are confirmed to be direct at $\Gamma$, and the difference between the DFT-PBE and HSE06 gaps provide us with the scissor operator that we used to correct the DFT-PBE gap for optical calculations.

In Figures $\mathrm{S} 3$ and $\mathrm{S} 4$, we show the band structures of all the experimentally measured structures calculated using the DFT-PBE exchange-correlation functional. We applied a kindependent and band-independent scissor operator to all the band structures according to Table S11, to match the band gap with that from HSE06 calculations. For all structures, we see that the DFT-PBE band structures with scissor operator match the HSE band structures reasonably well, thus the $\mathbf{k}$-independent and band-independent scissor operator approach is also used to obtain the optical spectra in the main text. This allows us to avoid expensive HSE06 calculations on dense k-point grids to provide the correction of DFT-PBE for the optical spectra. The density of states are normalized to per formula unit for all materials, with the formula units given in the captions.

Table S11: Comparison between the calculated band gaps (in eV) from DFT-PBE and HSE06, as well as experiments, for all the structures measured experimentally.

\begin{tabular}{c|cccc}
\hline & DFT & HSE & $\Delta_{\text {scissor }}$ & Expt. $E_{g}(\mathrm{eV})$ \\
\hline $\mathrm{Na}_{2} \mathrm{Zn}_{2} \mathrm{~S}_{3}$ & 2.15 & 3.26 & 1.11 & 3.6 \\
$\mathrm{Na}_{6} \mathrm{ZnSe}_{4}$ & 1.66 & 2.58 & 0.92 & 3.0 \\
$\mathrm{Na}_{2} \mathrm{ZnSe}_{2}$ & 1.80 & 2.76 & 0.97 & 2.9 \\
$\mathrm{Na}_{2} \mathrm{Zn}_{2} \mathrm{Se}_{3}$ & 1.47 & 2.47 & 0.99 & - \\
$\mathrm{K}_{6} \mathrm{ZnS}_{4}$ & 1.99 & 2.93 & 0.93 & - \\
$\mathrm{K}_{2} \mathrm{ZnS}_{2}$ & 2.61 & 3.68 & 1.07 & 3.9 \\
$\mathrm{~K}_{2} \mathrm{Zn}_{3} \mathrm{~S}_{4}$ & 2.55 & 3.66 & 1.11 & 3.7 \\
$\mathrm{~K}_{2} \mathrm{ZnSe}_{2}$ & 2.12 & 3.08 & 0.97 & 3.4 \\
$\mathrm{~K}_{2} \mathrm{Zn}_{3} \mathrm{Se}_{4}$ & 1.92 & 2.93 & 1.01 & 3.0 \\
$\mathrm{ZnS}$ & 2.00 & 3.22 & 1.22 & $3.5[7]$ \\
$\mathrm{ZnSe}$ & 1.13 & 2.23 & 1.11 & $2.6[7]$ \\
\hline
\end{tabular}


(a) $\mathrm{K}_{2} \mathrm{Zn}_{3} \mathrm{~S}_{4}$

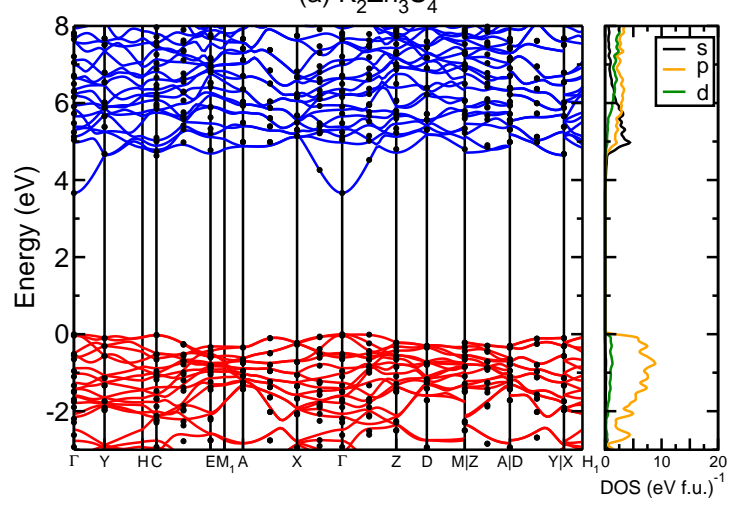

(c) $\mathrm{K}_{6} \mathrm{ZnS}_{4}$

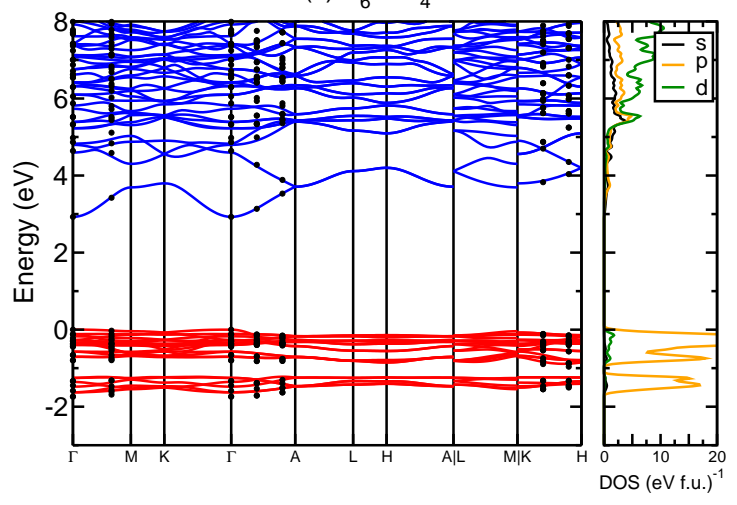

(b) $\mathrm{K}_{2} \mathrm{ZnS}_{2}$

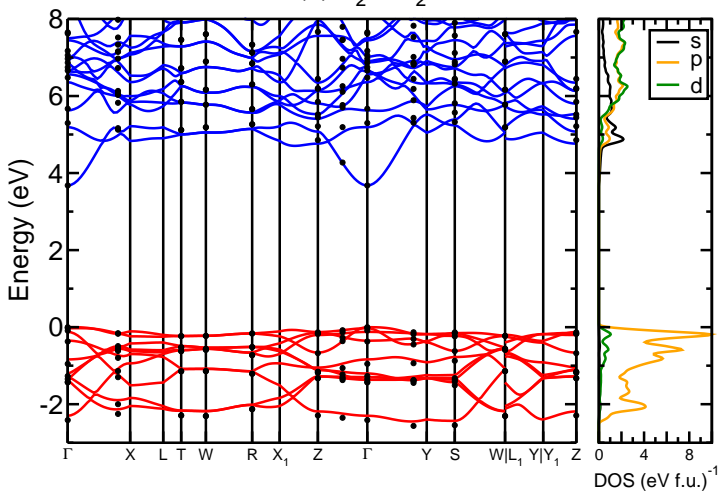

(d) $\mathrm{Na}_{2} \mathrm{Zn}_{2} \mathrm{~S}_{3}$

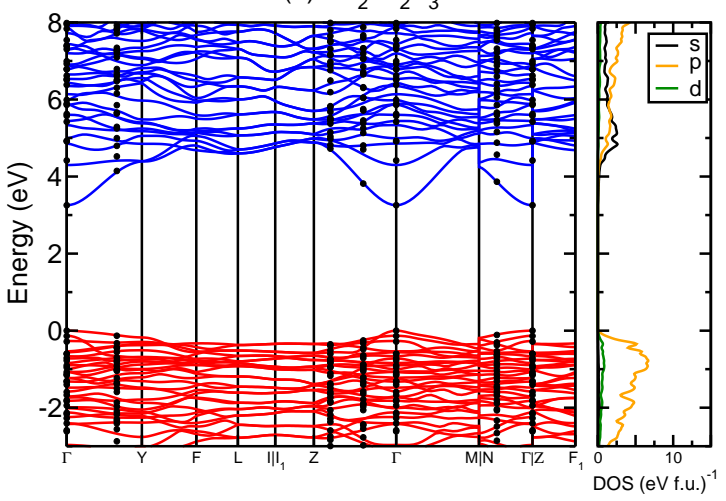

(e) $\mathrm{ZnS}$

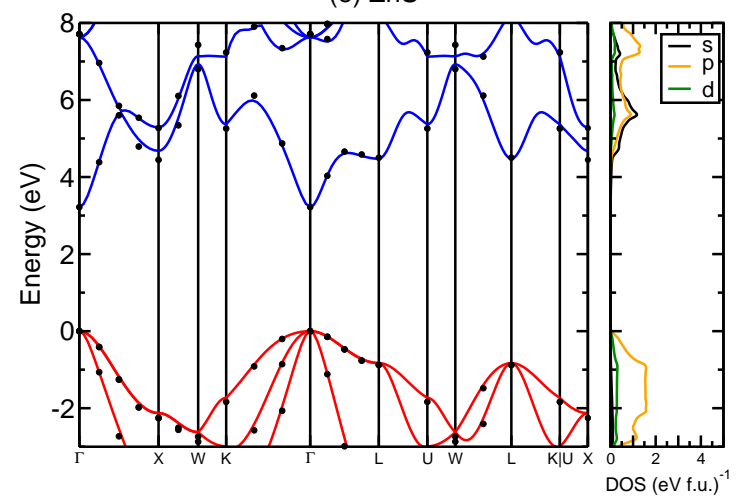

Figure S3: Electronic band structures and density of states for the sulfides calculated using DFT-PBE with scissor shift to match the HSE06 band gap. Black dots represents band structures from the HSE calculations. 

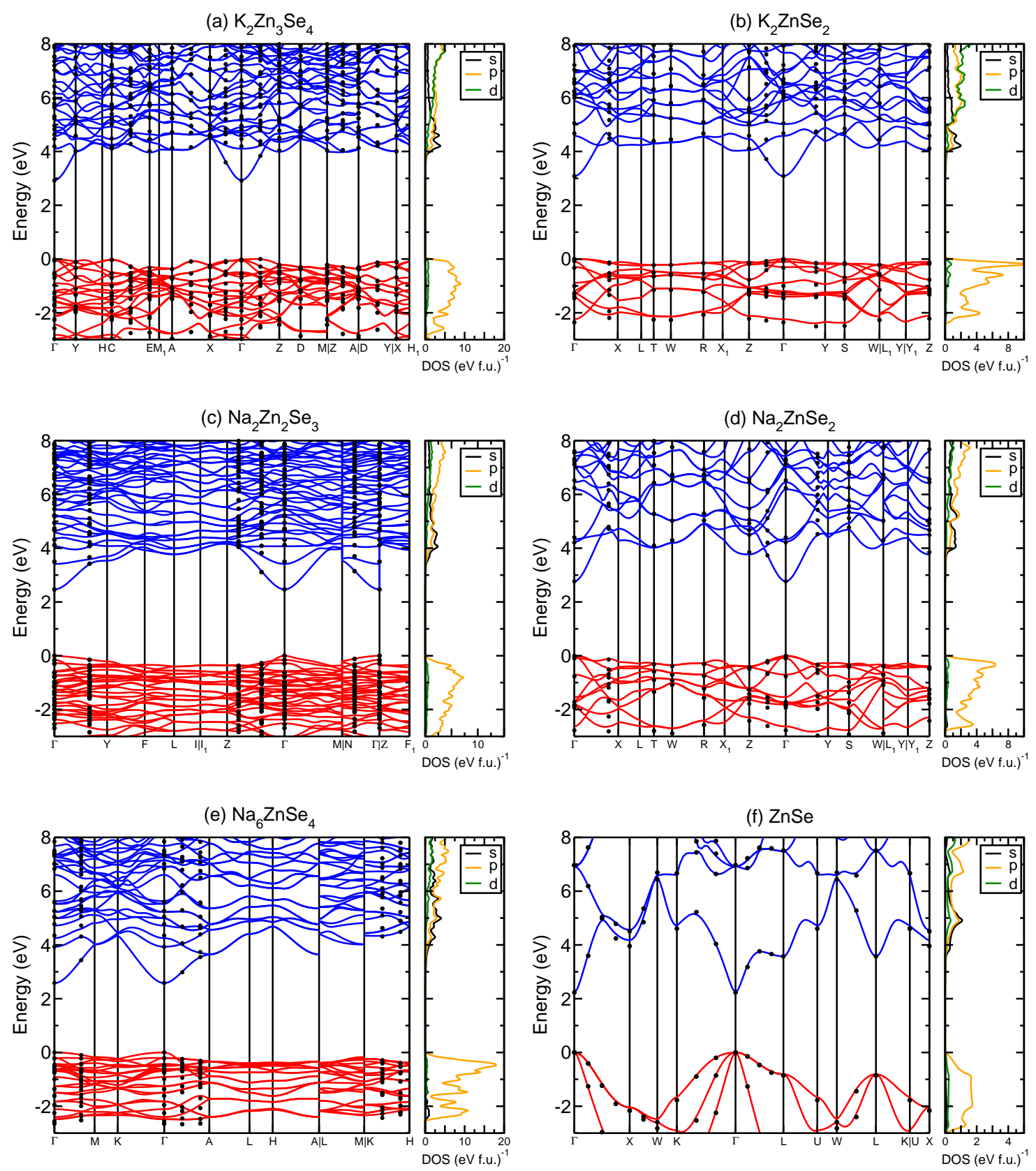

Figure S4: Electronic band structures and density of states for the selenides calculated using DFT-PBE with scissor shift to match the HSE06 band gap. Black dots represents band structures from the HSE calculations. 


\section{Optical properties}

We here provide the optical spectra calculated using the DFT-PBE exchange correlation functional, without considering electron-hole interactions, and using a scissor operator according to Table S11. As we can see from Fig. S5 and Fig. S6, most of the structures here show some degree of anisotropy, due to the pseudo-1D and pseudo-2D nature mentioned in the main text, however, more detailed studies using the BSE approach (with local-field effects) are unfeasible due to the large unit cells of these materials, except for the pseudo-1D $A_{2} \mathrm{Zn} Q_{2}$ structures. It can also be seen that the anisotropy in other structures is not as strong as in the pseudo 1-D $A_{2} \mathrm{Zn} Q_{2}$ structures, thus we did not perform BSE calculations explicitly for materials other than $A_{2} \mathrm{Zn} Q_{2}$.

In Fig. S7, we show the comparison of the imaginary part of the dielectric function with the scissor operator, and the calculated absorption coefficient of different compounds in the same K-Zn-S family. This provides information on how optical properties change with the $\mathrm{Zn}$ fraction. The absorption coefficient as a function of frequency is obtained through its relationship with the complex dielectric function [8]:

$$
\alpha(\omega)=2 \frac{\omega}{c} \operatorname{Im} \sqrt{\varepsilon(\omega)}
$$

Here $\alpha(\omega)$ is the absorption coefficient, $c$ is speed of light, and $\varepsilon(\omega)$ is the complex dielectric function. For all materials, the shape of the absorption onsets remains similar, $\sim 1 \mathrm{eV}$ above the band gap, except for the difference in the gap itself. The sharp increase of $\operatorname{Im} \varepsilon(\omega)$ is blue-shifted as the Zn fraction decreases, and as a result, the onset of strong UV absorption is blueshifted as the $\mathrm{Zn}$ fraction decreases. 

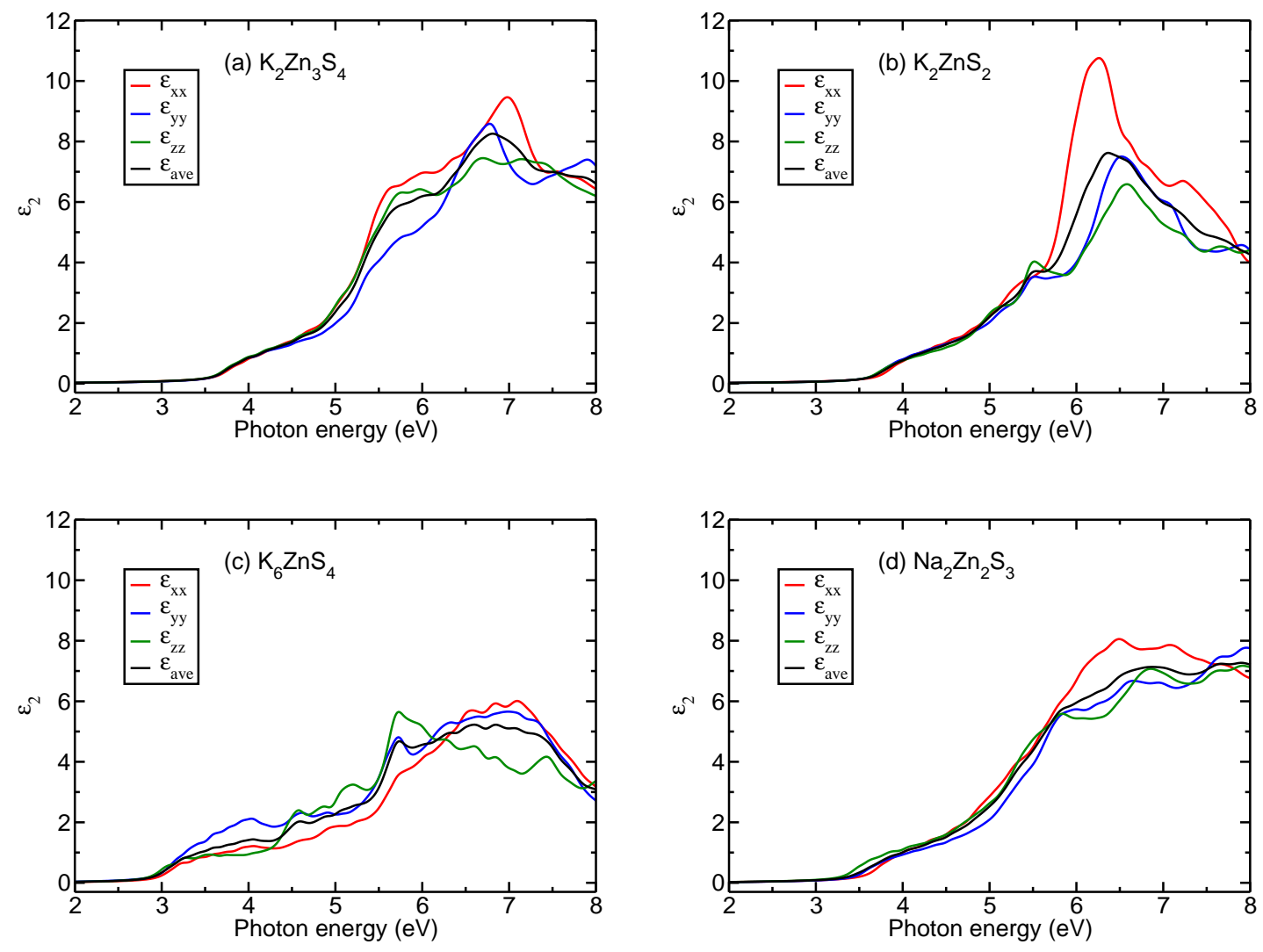

Figure S5: Optical spectra from independent particle approach for the experimentally measured sulfides. 

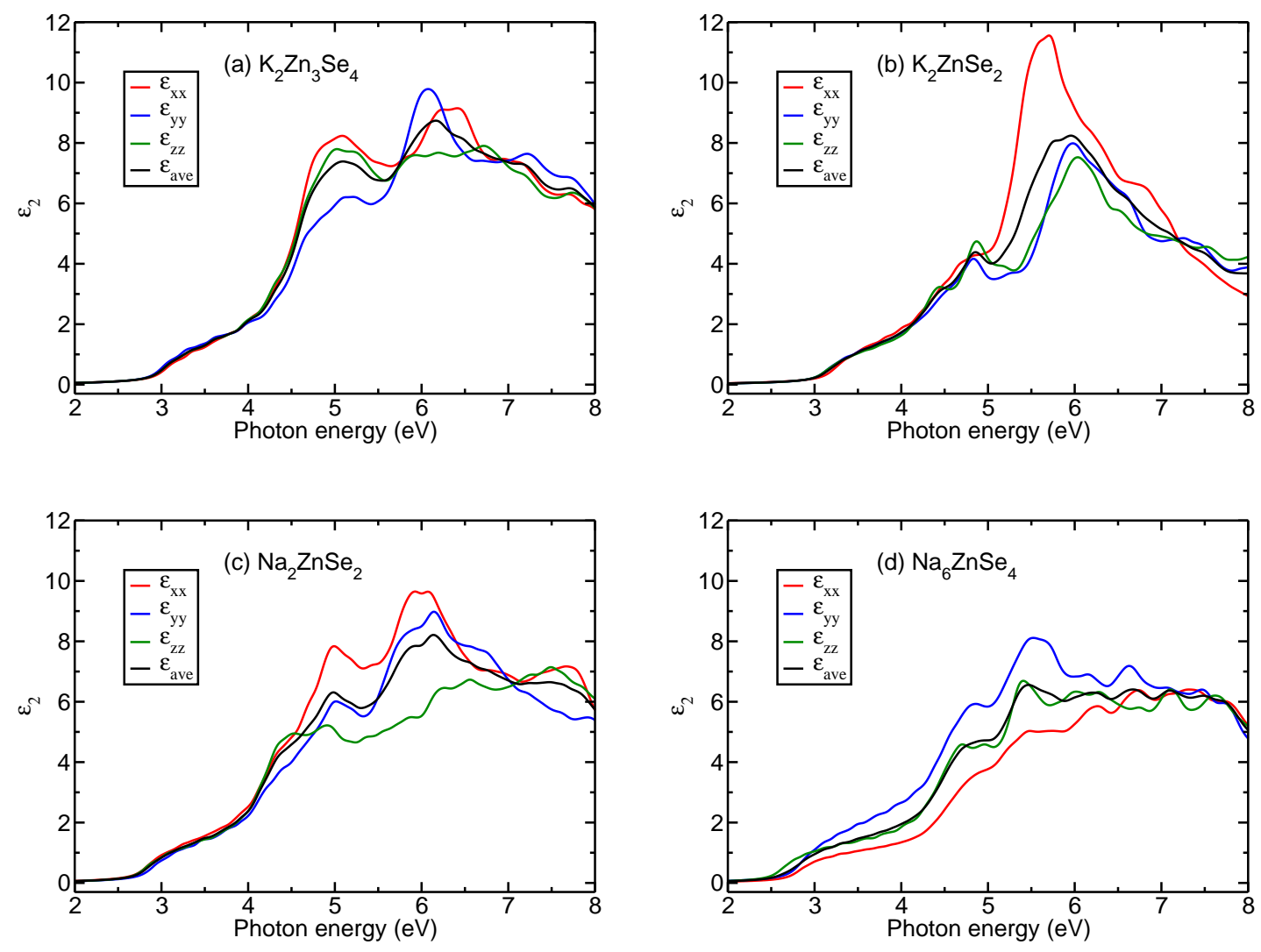

Figure S6: Optical spectra from independent particle approach for the experimentally measured selenides. 


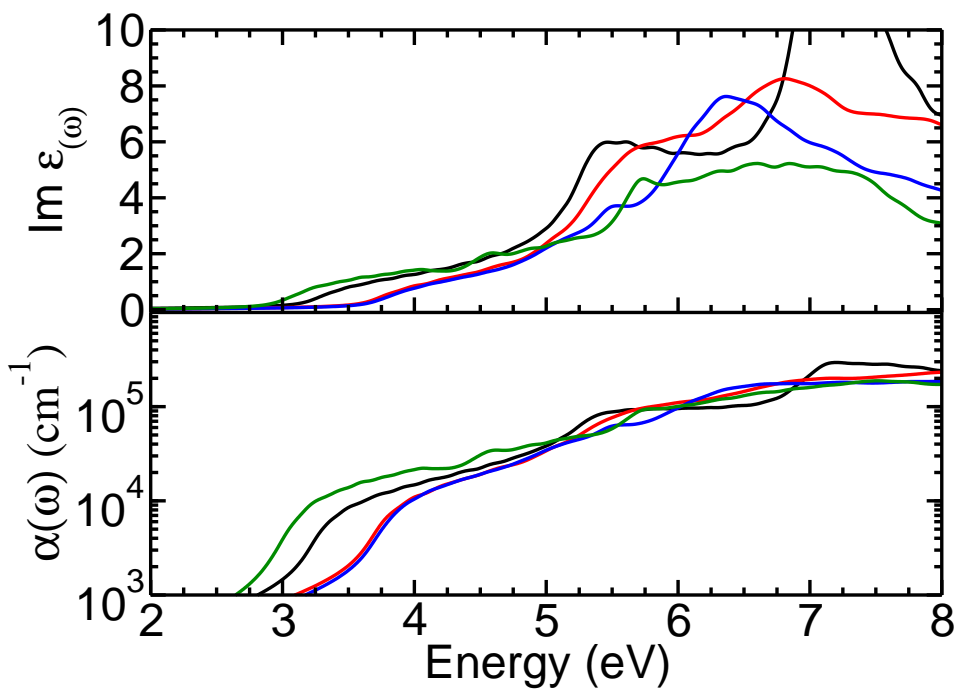

Figure S7: Upper panel: Imaginary part averaged over $x x, y y$, and $z z$ of the dielectric function calculated within the DFT-PBE exchange-correlation functional of the K-Zn-S family: $\mathrm{ZnS}$ (black), $\mathrm{K}_{2} \mathrm{Zn}_{3} \mathrm{~S}_{4}$ (red), $\mathrm{K}_{2} \mathrm{ZnS}_{2}$ (blue), and $\mathrm{K}_{6} \mathrm{ZnS}_{4}$ (green). Lower panel: the corresponding absorption coefficient. Scissor shift operator is applied to match the HSE06 band gap. 


\section{$6 \quad$ States-projected optical spectra}

In this section, we show optical spectra of $\mathrm{K}_{2} \mathrm{ZnS}_{2}$ and $\mathrm{K}_{2} \mathrm{ZnSe}_{2}$, decomposed according to transitions from $s, p$, and $d$ valence states of $\mathrm{K}, \mathrm{Zn}$, and $\mathrm{S} / \mathrm{Se}$ into all conduction bands, see Figs. $\mathrm{S} 8(\mathrm{a})$ and (c), as well as transitions from all valence bands into $s, p$, and $d$ conduction states of K, Zn, and S/Se, see Figs. S8(b) and (d). These spectra are calculated within DFT-PBE, applying the same scissor shift as discussed before, and averaged over the three directions of light polarization. The projected spectra are calculated, taking into consideration the projection $p_{l m n \mathbf{k}}^{I}$ of the wave function $\phi_{n \mathbf{k}}$ on to spherical harmonic $Y_{l m}$ for a given ion $I$, using [9]:

$$
\epsilon_{2}^{\alpha \beta}(\omega)=\frac{8 \pi^{2} e^{2}}{\Omega} \sum_{c, v, \mathbf{k}}\left(\sum_{l, m, I} p_{l m n \mathbf{k}}^{I}\right) \frac{\left\langle\phi_{c \mathbf{k}}\left|\hat{p}_{\alpha}\right| \phi_{v \mathbf{k}}\right\rangle\left\langle\phi_{v \mathbf{k}}\left|\hat{p}_{\beta}\right| \phi_{c \mathbf{k}}\right\rangle^{*}}{\left(E_{c \mathbf{k}}-E_{v \mathbf{k}}\right)^{2}} \delta\left(E_{c \mathbf{k}}-E_{v \mathbf{k}}-\hbar \omega\right)
$$

In the equation, $E_{n \mathbf{k}}(n=c, v)$ are the conduction band and valence band energies of wave vector $\mathbf{k}$, with the Kohn-Sham wave function $\phi_{n \mathbf{k}} \cdot \hat{p}$ is the momentum operator and $\Omega$ is the volume of the cell. For each electronic state $n \mathbf{k}$, the summation $\left(\sum_{l, m, I} p_{l m n \mathbf{k}}^{I}\right)$ equals one. Restricting this sum to only certain angular momentum components $l$ and/or certain ions $I$, the decomposed spectra in Fig. S8 are obtained. They show clearly that for both materials, the main peak between 6 and $7 \mathrm{eV}$ can be attributed to mainly transitions from $\mathrm{S} / \mathrm{Se} p$ states into all conduction bands. Our results also show that transitions into K $d$ (green solid), Zn $s$ (red dotted), and S/Se $p$ (blue dashed) states from all valence states form this main peak. 

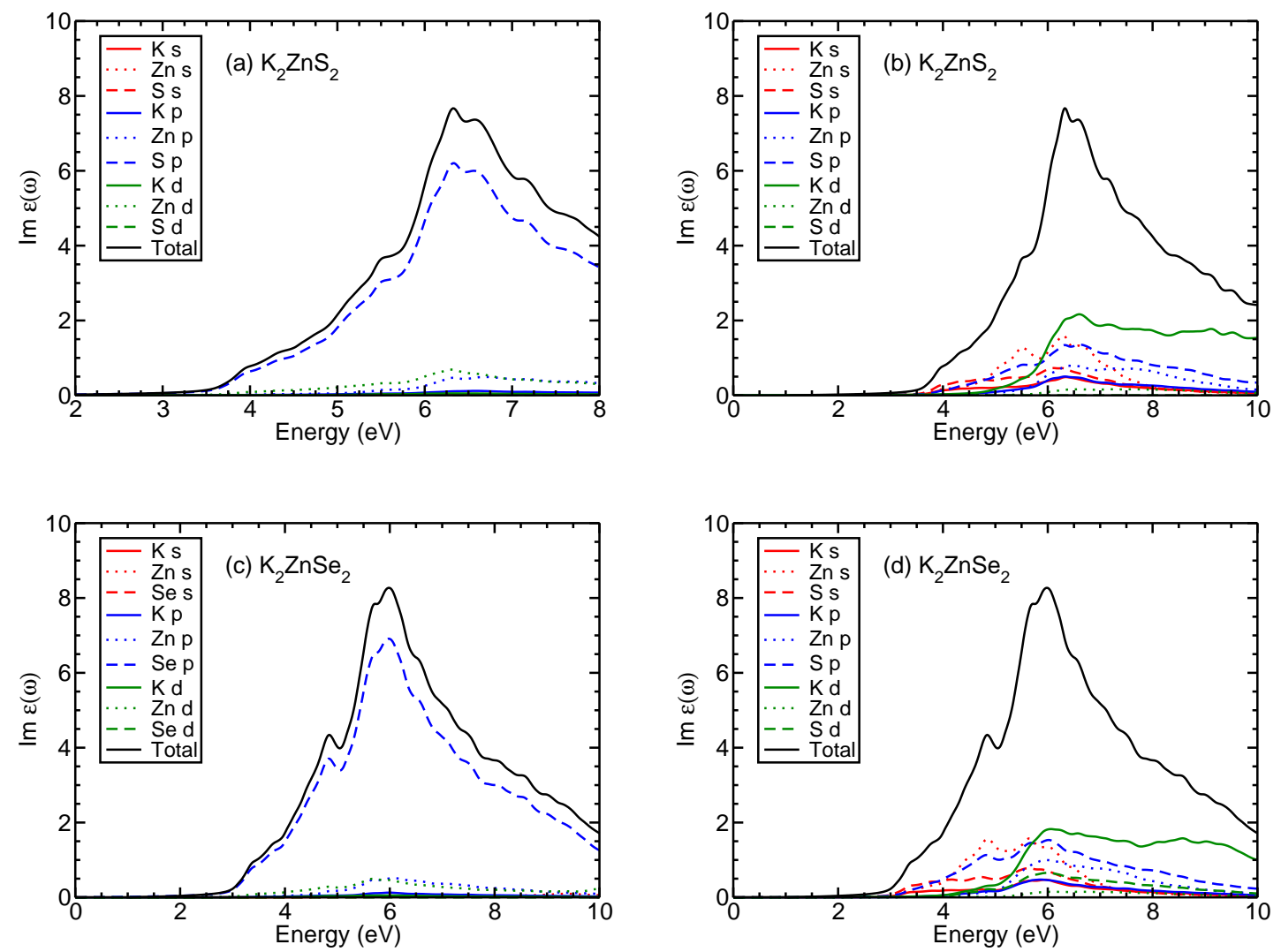

Figure S8: Imaginary part of the dielectric function, averaged over all light polarization directions. Spectra are resolved regarding character of their initial (a, c) or final (b, d) states. We show transitions from: (a) $s, p$, and $d$ valence states of $\mathrm{K}$, $\mathrm{Zn}$, and $\mathrm{S}$ into all conduction bands of $\mathrm{K}_{2} \mathrm{ZnS}_{2}$. (b) all valence bands into $s, p$, and $d$ conduction states of $\mathrm{K}$, $\mathrm{Zn}$, and $\mathrm{S}$ of $\mathrm{K}_{2} \mathrm{ZnS}_{2}$. (c) $s, p$, and $d$ valence states of $\mathrm{K}, \mathrm{Zn}$, and Se into all conduction bands of $\mathrm{K}_{2} \mathrm{ZnSe}_{2}$. (d) all valence bands into $s, p$, and $d$ conduction states of $\mathrm{K}$, Zn, and Se of $\mathrm{K}_{2} \mathrm{ZnSe}_{2}$. Black curves show spectra summed over all contributions. 


\section{Real part of the dielectric function}

In this section, we show the calculated real part of the dielectric functions, obtained through the Kramers-Kronig relation from the imaginary part of the dielectric functions. In Fig. S9 and Fig. S10, we show the DFT-PBE results and in Fig. S11, we show the BSE results for $\mathrm{K}_{2} \mathrm{ZnS}_{2}, \mathrm{~K}_{2} \mathrm{ZnSe}_{2}$, and $\mathrm{Na}_{2} \mathrm{ZnSe}_{2}$.
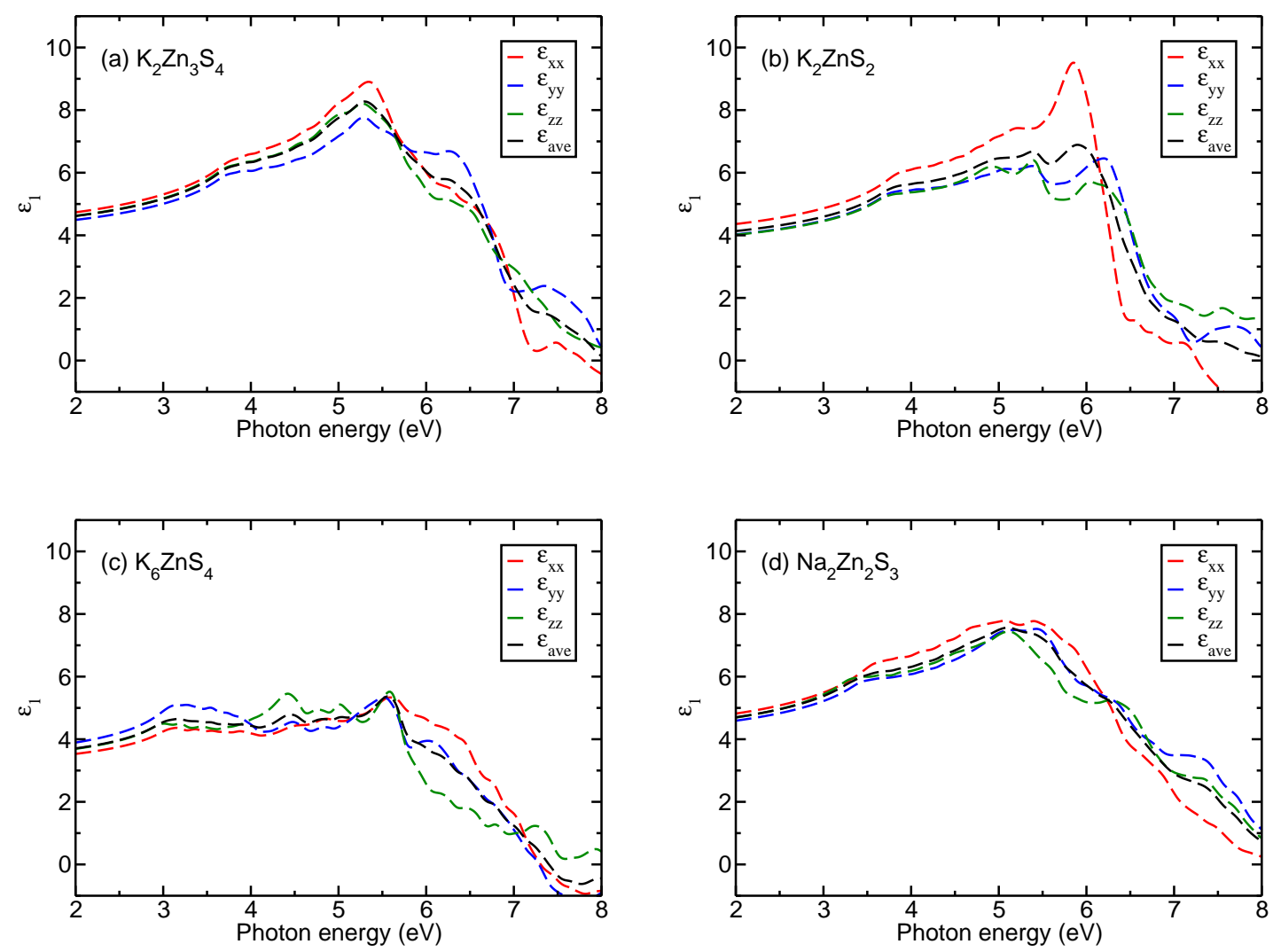

Figure S9: Real part of the dielectric functions, computed using the independent particle approach, for the experimentally measured sulfides. 

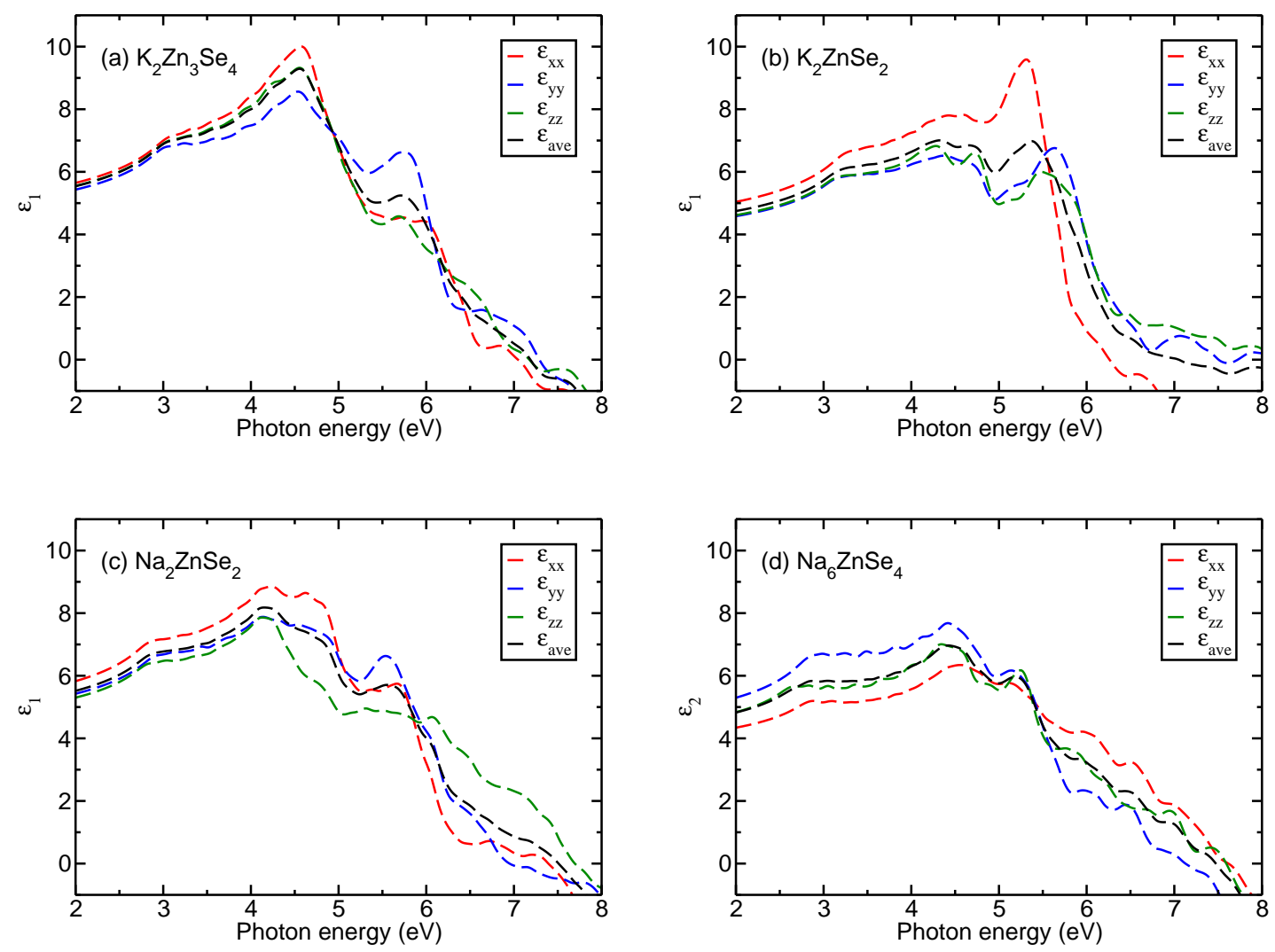

Figure S10: Real part of the dielectric functions, computed using the independent particle approach, for the experimentally measured selenides. 

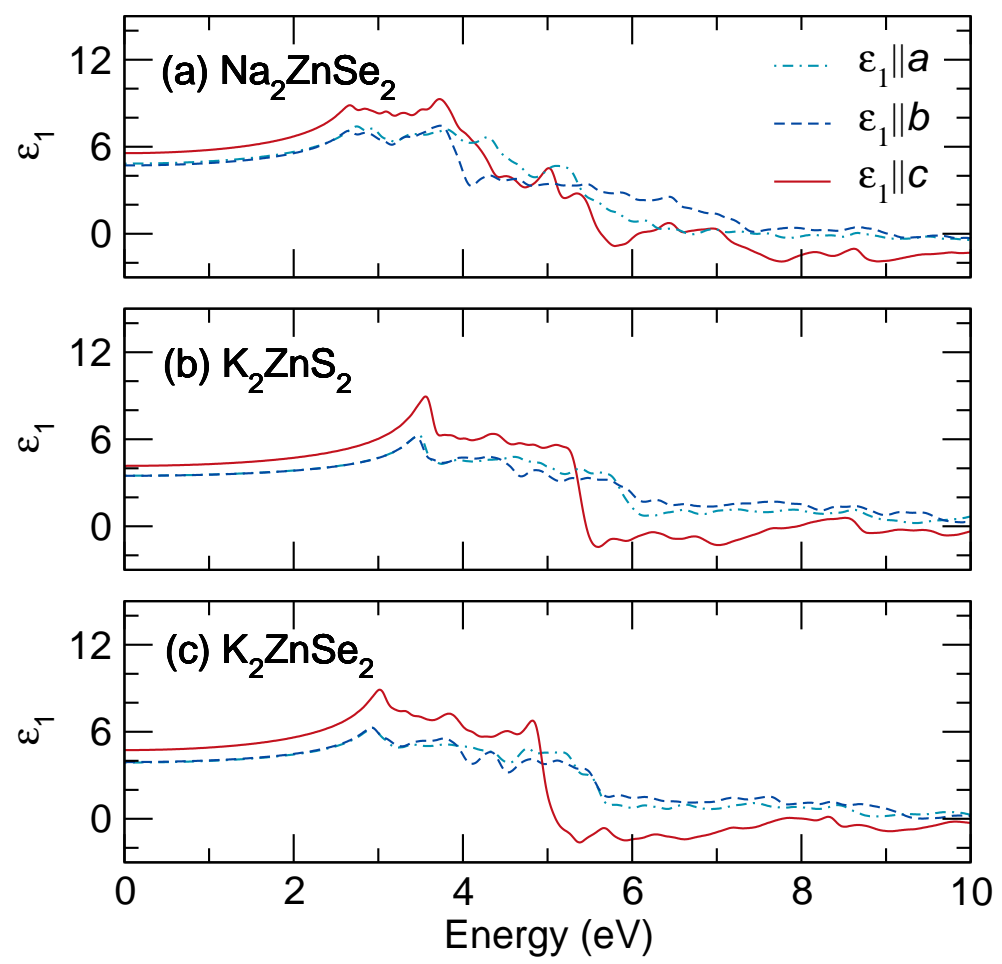

Figure S11: Real part of the dielectric functions, calculated with the inclusion of excitonic effects using the BSE approach, for (a) $\mathrm{Na}_{2} \mathrm{ZnSe}_{2}$, (b) $\mathrm{K}_{2} \mathrm{ZnS}_{2}$, and (c) $\mathrm{K}_{2} \mathrm{ZnSe}_{2}$. 


\section{References}

[1] G Hautier, C Fischer, V Ehrlacher, A Jain, and G Ceder. Data mined ionic substitutions for the discovery of new compounds. Inorg. Chem., 50(2):656-663, 2010.

[2] A Jain, S P Ong, G Hautier, W Chen, W D Richards, S Dacek, S Cholia, D Gunter, D Skinner, G Ceder, and K A Persson. The Materials Project: A materials genome approach to accelerating materials innovation. APL Materials, 1(1):011002, 2013.

[3] W Setyawan and S Curtarolo. High-throughput electronic band structure calculations: Challenges and tools. Comput. Mater. Sci., 49(2):299-312, 2010.

[4] S Curtarolo, W Setyawan, G L W Hart, M Jahnatek, R V Chepulskii, R H Taylor, S Wang, J Xue, K Yang, O Levy, et al. Aflow: an automatic framework for highthroughput materials discovery. Comput. Mater. Sci., 58:218-226, 2012.

[5] J Heyd, G E Scuseria, and M Ernzerhof. Erratum: Hybrid functionals based on a screened coulomb potential? [J. Chem. Phys. 118, 8207 (2003)]. J. Chem. Phys., 124(21):219906, 2006.

[6] J Heyd, G E Scuseria, and M Ernzerhof. Hybrid functionals based on a screened coulomb potential. J. Chem. Phys., 118(18):8207-8215, 2003.

[7] T Homann, U Hotje, M Binnewies, A Börger, K-D Becker, and T Bredow. Compositiondependent band gap in $\mathrm{ZnS}_{x} \mathrm{Se}_{1-x}$ : a combined experimental and theoretical study. Solid State Sci., 8(1):44-49, 2006.

[8] C F Klingshirn. Semiconductor optics. Springer Science \& Business Media, 2012.

[9] J Leveillee, C Katan, L Zhou, A D Mohite, J Even, S Tretiak, A Schleife, and A J Neukirch. Influence of $\pi$-conjugated cations and halogen substitution on the optoelectronic and excitonic properties of layered hybrid perovskites. Phys. Rev. Mater., 2(10):105406, 2018. 